\title{
Evaluations of the Effect of Sodium Metabisulphite on the Stability and Dissolution Rates of Various Model Drugs from the Extended Release Polyethylene Oxide Matrices
}

\author{
Saeed Shojaee $^{1}$ - Ali Nokhodchi ${ }^{2,3} \cdot$ Mohammed Maniruzzaman $^{2}$
}

Published online: 15 May 2017

(C) The Author(s) 2017. This article is an open access publication

\begin{abstract}
Purpose This study examines the effect of sodium metabisulphite (SMB) as an antioxidant on the stability and release of various model drugs, namely, propranolol $\mathrm{HCl}$, theophylline and zonisamide from the polyethylene oxide (PEO) tablets. The antioxidant was used to minimise degradation and instability of the manufactured tablets when stored at $40{ }^{\circ} \mathrm{C}$ $(55 \pm 5 \% \mathrm{RH})$ over 8 weeks.

Method Multiple batches of tablets weighing $240 \mathrm{mg}(50 \% \mathrm{w} /$ $w)$ with a ratio of $1: 1 \mathrm{drug} /$ polymer and $1 \%(w / w)$ sodium metabisulphite containing different model drugs and various molecular weights of PEO 750 and 303 were produced.

Results The results indicated that the use of sodium metabisulphite marginally assisted in reducing drug release and degradation via oxidation in propranolol $\mathrm{HCl}$ tablets containing both PEO 750 and 303. In the case of poorly and semisoluble drugs (zonisamide and theophylline), the formulations with both PEO showed entirely superimposable phenomenon
\end{abstract}

Ali Nokhodchi

A.Nokhodchi@sussex.ac.uk

$\triangle$ Mohammed Maniruzzaman

M.Maniruzzaman@sussex.ac.uk; M.Maniruzzaman12@gmail.com

Saeed Shojaee

shojaee41@yahoo.com

1 Medway School of Pharmacy, University of Kent, Anson Building, ME4 4TB Chatham, UK

2 Department of Pharmacy (Chemistry), School of Life Sciences, University of Sussex, Falmer, BN1 9QJ Brighton, UK

3 Drug Applied Research Center and Faculty of Pharmacy, Tabriz University of Medical Sciences, Tabriz, Iran and different release profiles compared to control samples (matrices without SMB). DSC study demonstrated the modifications of the polymer due to degradation and observed the effect of SMB on the thermal degradation of the PEO matrices.

Conclusion The use of antioxidant has assisted in retaining the stability of the manufactured tablets with different model drugs especially those with the highly soluble drug that are susceptible to rapid degradation. This has been reflected by an extended release profile of various drugs used at various stages of the storage time up to 8 weeks.

Keywords Sodium metabisulphite (SMB) - Antioxidant . Dissolution rates $\cdot \mathrm{PEO} \cdot$ Molecular weight $\cdot$ Matrix tablets

\section{Introduction}

Chemical degradation may affect the physical appearance and dissolution rates, which are important factors relating to the stability of the manufactured tablets. The chemical degradation of the active substance from the manufactured tablets can reduce the amount of drug available to the patient, making it questionable by the regulatory bodies. Among various factors, namely, temperature, solvent, $\mathrm{pH}$, light and oxygen, are some examples that can affect the drug stability. In addition, excipients can cause stability problems as well due to their direct chemical reactions with the drug molecules $[1,2]$. Hydrophilic matrix technology is frequently used to develop controlled release drug delivery systems [3] as it provides convenient release profiles for a wide range of therapeutic drugs and enhances the patient compliances [4]. It has been reported that a robust formulation strategy makes it easy to 
manufacture at an economical cost with a good in vivo and in vitro correlation $[5,6]$. The hydrophilic polymers commonly used to develop controlled release formulations initiate a prompt and consistent hydration resulting in the formation of the gel layer that helps retain structural integrity and inhibits tablet disintegration $[7,8]$.

The outer layer allows for the disentanglement of the polymer from the surface of the matrix when it eventually reaches a dilution point due to its highly hydrated nature $[4,9,10]$. The gel layer is then continually replaced with the hydrated polymer from inside the core. Lapidus and Lordi (1966) states, "the release of a drug from the hydrophilic matrix relies on swelling of the matrix, dissolution of the drug, and diffusion and erosion properties of the gel layer" [11]. However, the mechanism of drug release is influenced by both the solubility and dose of the drug, type and quantity of fillers and the polymer $[12,13]$. The solubility of the tablet composition is important, as it dictates the rate and extent of drug release from the tablets as well as the polymer hydration and swelling [4, 14-17].

Recently, polyethylene oxide (PEO) has been used as one of the most important materials in the pharmaceutical industry mainly because of its low toxicity, availability in a range of molecular weight/viscosity grades, wide regulatory acceptances and insensitivity to the $\mathrm{pH}$ of the biological medium, high water swellability and erosion characteristics [18-21]. It has been reported that the molecular weight of the matrix determines the release rate of the model drug [19, 22, 23]. A study [1, 24], outlined that the higher molecular weight PEO involves a combination of swelling and diffusion whereas the lower molecular weight PEO inhibits a swelling and erosion as the main mechanism of drug release. Sodium metabisulphite is a crystalline powder that is readily soluble in water. It is mainly used as a preservative in some drug and food preparation because of its anti-oxidant properties. It acts as an oxygen scavenger to eliminate dissolved oxygen from water. It is stable under normal conditions and is hygroscopic in nature $[25,26]$.

Oxidative degradation is highly likely as PEO can undergo an autoxidation resulting in the degradation of the formulations. The use of antioxidant can eliminate degradation to a large extent, thus controlling the unexpected release of the drug as a result of the degradation of the carrier matrices. This research examined the effect of sodium metabisulphite (SMB) as an antioxidant on the stability of PEO 750 and 303 tablet formulations containing various soluble model drugs as well as their release rates. The effect of SMB to control the release rates of various model drugs as a function to minimise the degradation and instability of PEO matrix tablets when stored at $40{ }^{\circ} \mathrm{C}(55 \pm 5 \% \mathrm{RH})$ for 8 weeks was explored.

\section{Materials and Methods}

\section{Materials}

Propranolol $\mathrm{HCl}$, theophylline, zonisamide and sodium metabisulphite $(>97 \%)$ (MW $=190.10)$ were provided by Acros organics (Geel, Belgium). Sentry polyox WSR 750 (MW 300,000), LEO NF and Sentry polyox WSR 303 $(\mathrm{MW}=7,000,000)$, LEO NF, were kindly donated by Colorcon Ltd. (Dartford, Kent, UK). Magnesium stearate (MgSt) was purchased from Fischer Scientific (Loughborough, UK). Deionised water was supplied by Medway School of Pharmacy (Kent, UK).

\section{True Density Measurement of Powders}

In order to evaluate any changes in the true density of powders before and after storage times (0, 2, 4 and 8 weeks), an ultrapycnometer 1000 (Qunatochrom, USA) was used. To carry out this test, about 3-5 g of sample was used and the results are the mean and standard deviation of three determinations. Tablet dimensions were obtained using an electronic digital calliper (Fisher Scientific, Loughborough, UK).

\section{Tablet Preparation}

Multiple batches of tablets weighing $240 \mathrm{mg}$ with a drug/polymer $1: 1$ ratio $(50: 50 \% w / w)$ containing different model drugs and various molecular weights of polyox 750 and 303 were prepared. Batches also contained $1 \%$ w/w sodium metabisulphite as an antioxidant. All formulations were mixed in a Turbula system manufactured by Schatz Willy A (Basel, Switzerland) for a period of $10 \mathrm{~min}$. Tablet matrices with a target weight of $240 \mathrm{mg}$ were prepared using a manual tablet press produced by Enerpac (Northumberland, UK), equipped with an 8-mm flat-faced die and punch set, at a compression pressure of $1500 \mathrm{psi}$. The dye and punch were lubricated with a $1 \% \mathrm{w} / \mathrm{v}$ mixture of magnesium stearate and acetone. The tablets were stored in a covered close glass container in an oven at $40{ }^{\circ} \mathrm{C}(55 \pm 5 \% \mathrm{RH})$. At different time intervals $(0,2,4$ and 8 weeks), the release rate of the tablets was determined from dissolution tests.

\section{Physical Characterisation of Tablets}

The crushing strength (hardness) test $(n=3)$ was carried out using a hardness tester DR SCHLEUNIGER obtained from JB Pharmatron Ltd. (Northamptonshire, UK). The averages of these three tablets were calculated as mean results. 


\section{Dissolution Study}

In vitro drug release from matrix tablets was carried out using the USP paddle method [27]. The dissolution tester manufactured by Erweka (Heusenstamm, Germany) was used to monitor the dissolution profiles of the model drugs in the matrix tablets. The dissolution medium consisted of $900 \mathrm{ml}$ of distilled water at $37 \pm 0.1{ }^{\circ} \mathrm{C}$, and the paddles were rotated at $100 \mathrm{rpm}$. The sink condition was maintained for all the three drugs used in the present study according to their solubility in the water $(0.8,13$ and $50 \mathrm{mg} / \mathrm{ml}$ for zonisamide, theophylline and propranolol $\mathrm{HCl}$, respectively), and the dose used in the present study was $120 \mathrm{mg}$ for each drug. From the dissolution flask, samples were withdrawn at preselected time intervals at 15-min intervals up to $2 \mathrm{~h}$ and after that, every $30 \mathrm{~min}$ up to $12 \mathrm{~h}$ using a peristaltic pump. The concentrations of drugs in the samples were determined by UV spectrophotometer at 271, 270 and $290 \mathrm{~nm}$ for theophylline, zonisamide and propranolol $\mathrm{HCl}$, respectively, using UV-visible recording spectrophotometer (Shimadzu, Kyoto, Japan).

\section{Kinetics Models}

In order to study the mechanism of drug release from matrix tablets, the release data were fitted to well-known empirical equation proposed by Korsmeyer and Peppas [28].

$M_{t} / M_{\infty}=K_{p} t^{n}$

$\log M_{t} / M_{\infty}=\log K_{p}+n \log t$

where $M_{t} / M$ is the fractional drug release, $t$ is the release time, $K_{p}$ denotes as the kinetic constant and $n$ is the diffusion exponent characteristics of the release mechanism. For a cylinder matrix that can swell, $0.89<n<1$ indicates a super case II, and $n=0.89$ shows for the case II release kinetics, while $0.45<n<0.89$ shows anomalous release kinetics and when $n<0.45$ shows Fickian diffusion release kinetics [28, 29].

\section{Similarity Factor $\left(f_{2}\right)$ Measurement}

To determine the similarity between the obtained drug release profiles, the $f_{2}$ factor proposed by Moore and Flanner [30] was calculated according to Eq. (1):

$f_{2}=50 \log \left\{\left[1+1 / \sum_{n-1}^{n} w t\left(R_{t}-T_{t}\right)^{2}\right]^{-0.5} \times 100\right\}$

This being a mathematical treatment of the dissolution data, where $n$ is the number of test points for the samples, $w t$ is the optional weight factor, $R_{t}$ is the reference assay at time point $t$ and $T_{t}$ is the test assay at time point $t$. An $f_{2}$ value between 50 and 100 suggests a similarity between the two release profiles, and the closer the value is to 100 , the more similar or identical the profiles are. Also, dissimilarity occurs with decreasing values less than 50 [30].

\section{Dissolution Parameters}

Dissolution efficiency ( $\left.\mathrm{DE}_{720 \mathrm{~min}}\right)$ and mean dissolution time (MDT) were used to represent the dissolution rate from various preparations. The DE of a pharmaceutical dosage forms is defined as the area under the dissolution curve up to a certain time, $t$, expressed as percentage of the area of the rectangle described by $100 \%$ dissolution in the same time. DE is described by Eq. (4) [31]:

$\mathrm{DE}=\frac{\int_{0}^{t} y \times d t}{y_{100} \times t} \times 100 \%$

where $y$ is the percent drug release as the function of time, $t$ is the total time of drug release and $y_{100}$ is $100 \%$ drug release. An alternative parameter that describes the dissolution rate is the mean dissolution time (MDT), the most likely time for a molecule to be dissolved from a solid dosage form. Therefore, MDT is the mean time for the drug to dissolve under in vitro dissolution conditions. This is calculated using Eq. (5) [31]:

$\mathrm{MDT}=\frac{\sum_{j-1}^{n} t_{j} \Delta M_{j}}{\sum_{j-1}^{n} \Delta M_{j}}$

where $j$ is the sample number, $t_{j}$ is the midpoint of the $j$ th time period (easily calculated with $[t+(t-1)] / 2$ ) and $\Delta M_{j}$ is the additional amount of drug dissolved between $t_{j}$ and $t-1$. SPSS software was then used to calculate the cumulative percentages of DE and MDT.

\section{Differential Scanning Calorimetry (DSC)}

DSC was used to characterise the thermal properties of the polymers. Metter Toledo DSC (Beaumont Leys, Leicester, UK) system TS0801RO sample robot was used to measure thermal properties (enthalpy) and melting point of the polymer, in the physical mixture. Approximately 4-5 mg of sample was weighed and heated ranging from 25 to $280{ }^{\circ} \mathrm{C}$ at a scanning rate of $10{ }^{\circ} \mathrm{C} / \mathrm{min}$ in sealed no thematic aluminium pans for 28 min under nitrogen gas.

\section{Statistical Analysis}

The samples were compared with the reference (control) by an analysis of variance (ANOVA), following Dunnet's test. The SPSS software was used to perform all statistical analyses. The $P$ value $<0.05$ was considered as significant. All the results are expressed as mean \pm standard deviation. 


\section{Results and Discussion}

\section{The Influence of Storage Conditions on True Density}

In order to investigate any changes in true density over storage condition, the true density measurement of matrices containing sodium metabisulphite was carried out for all formulations. The results indicated that there was no significant difference $(p>0.05)$ on the true densities of PEO polymer formulations in presence of sodium metabisulphite stored over 8 weeks at $40{ }^{\circ} \mathrm{C}$. The results showed that the range of true density for fresh samples was between 1.29 and $1.33 \mathrm{~g} / \mathrm{cm}^{3}$. As been claimed that there was no significant change observed in the true density of polymer formulations stored over 8 weeks, the values of the true density remained approximately the same $\left(1.27-1.30 \mathrm{~g} / \mathrm{cm}^{3}\right)$ as the fresh samples.

\section{The Influence of Sodium Metabisulphite on Hardness of Various Drug Tablet Matrices}

Table 1 shows the effect of sodium metabisulphite at a $1 \%(w /$ w) concentration on the hardness of propranolol $\mathrm{HCl}$, theophylline and zonisamide tablet matrices before and after storage time. In general, the hardness of the tablets increases as the molecular weight of the polymer increases. Interestingly, as shown in Table 1, there was no significant difference between the hardness of neither the fresh and aged (up to 4 weeks of storage) propranolol $\mathrm{HCl}$ matrices containing the antioxidant nor tablets prepared with different molecular weight PEO. It has only been seen that the hardness of the propranolol tablets was reduced slightly after 8 weeks of storage. This was due to the presence of the antioxidant that was preventing the possible degradation of the PEO matrices resulting in almost similar hardness. For comparison, the manufactured tablets without the SMB exhibited slightly lower values in the hardness of the tablets with PEO 750 (time $0=\sim 90.2 \mathrm{~N}$ and week $4=\sim 88.3 \mathrm{~N}$ ). In contrast, overall results indicate an increase in tablet hardness over time for semi- and poorly soluble drugs (theophylline and zonisamide). The molecular weights of the polymer matrices seemed to play a pivotal role to the increase in the hardness of the produced tablets. This could be attributed to the fact that the presence of SMB might reduce the bonding strength between particles in the matrices at week 0 , which then continues to increase as the storage time increases over 8 weeks.

\section{The Evaluation Effect of Sodium Metabisulphite at Ratio $(1 \% w / w)$ on Release Rate Behaviour of Propranolol HCl Tablet Matrices}

In order to evaluate the effect of sodium metabisulphite on drug release, propranolol was chosen as a model drug due to its high solubility in water (solubility of propranolol $\mathrm{HCl}$ is $50.0 \mathrm{mg} / \mathrm{ml}$ ) and results are shown in Fig. 1. The dissolution profile indicates that there were no significant differences between the release rates of the tablets at different storage time for both molecular weight PEO (compare Fig. 1a with $b$ and c). The results showed that the storage temperature sustained the drug release process from the matrices from 0 week to 8 weeks compared to that of the 'control' formulations (without SMB). As can be seen from Fig. 1 b, c drug release was controlled for different storage time (2, 4, 8 weeks) when sodium metabisulphite was incorporated in propranolol $\mathrm{HCl}$ polymer formulations. This can be attributed to an increasing in viscosity of gel layer and, the formation of a gel layer with a longer diffusional path with a rise in the concentration of PEO. This means degradation as results of storage did not happen in matrices in presence of this antioxidant. This could be depending on the effect of sodium metabisulphite which dilated penetration of oxygen into the tablet matrices resulting to no degradation, and a loss in molecular weight of PEO did not occur in PEO. These results complement the previous study carried out by Shojaee et al. [32] who investigated the effect of different concentrations SMB on release rate of diltiazem $\mathrm{HCl}$ tablet matrices. The results showed that the drug release was controlled and much slower in the presence of this antioxidant
Table 1 Effect of sodium metabisulphite on propranolol/ theophylline/zonisamide-polyox tablet hardness stored at $40{ }^{\circ} \mathrm{C}$

\begin{tabular}{llllc}
\hline PEO (grade) & Time (weeks) & $\begin{array}{l}\text { Hardness (N) } \\
1 \% \text { SMB propanolol }\end{array}$ & $\begin{array}{l}\text { Hardness (N) } \\
\text { 1\% SMB theophylline }\end{array}$ & $\begin{array}{l}\text { Hardness (N) } \\
1 \% \text { SMB zonisamide }\end{array}$ \\
\hline 750 & 0 & $92.0 \pm 0.3$ & $74.3 \pm 0.6$ & $78.0 \pm 0.1$ \\
750 & 2 & $92.1 \pm 0.2$ & $77.5 \pm 0.1$ & $80.0 \pm 1.0$ \\
750 & 4 & $92.0 \pm 0.1$ & $81.0 \pm 0.7$ & $83.5 \pm 0.5$ \\
750 & 8 & $91.5 \pm 0.5$ & $85.5 \pm 0.3$ & $85.0 \pm 0.2$ \\
303 & 0 & $98.5 \pm 0.5$ & $89.5 \pm 0.2$ & $94.0 \pm 0.1$ \\
303 & 2 & $98.2 \pm 0.3$ & $91.0 \pm 0.5$ & $96.1 \pm 0.5$ \\
303 & 4 & $98.0 \pm 0.6$ & $92.0 \pm 0.4$ & $99.0 \pm 0.2$ \\
303 & 8 & $97.4 \pm 0.4$ & $93.5 \pm 0.6$ & $101.5 \pm 0.1$ \\
\hline
\end{tabular}



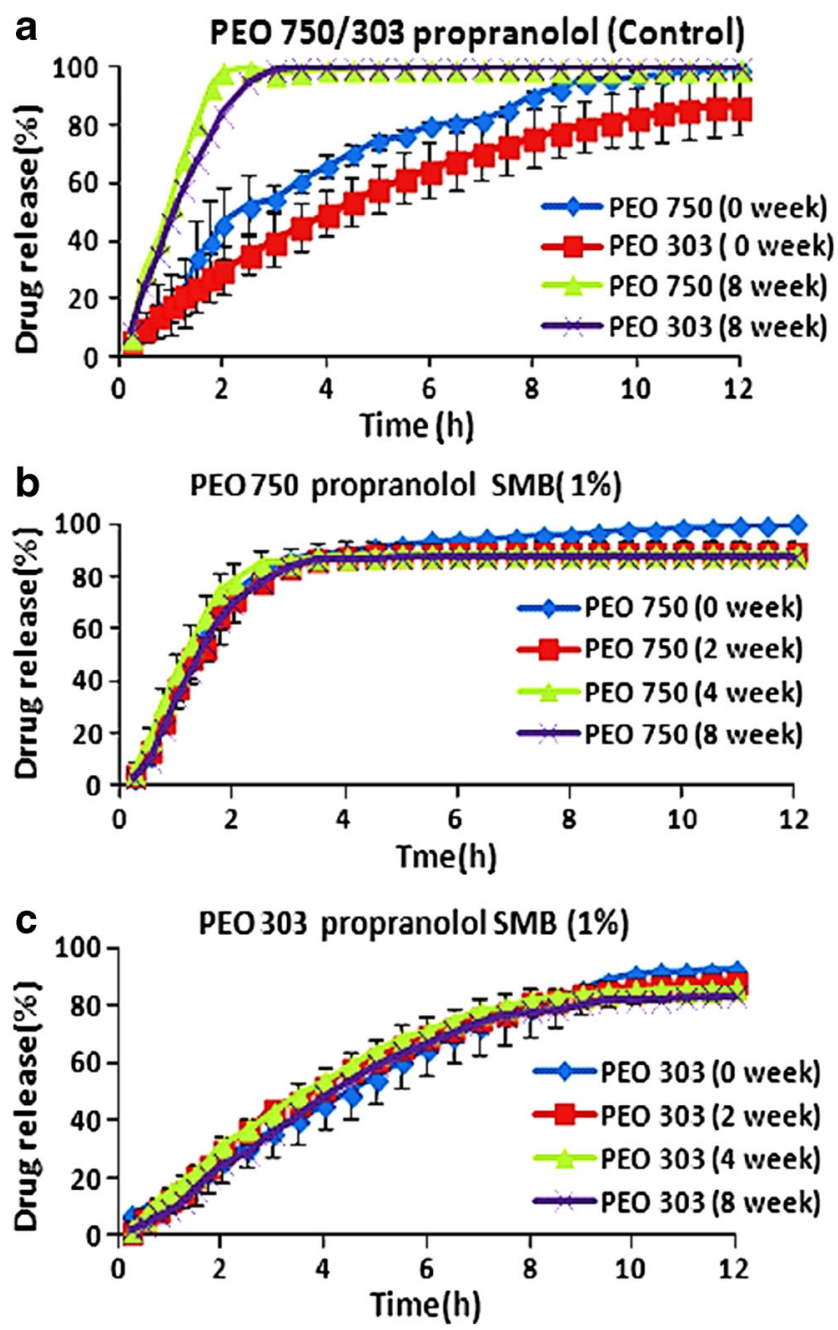

Fig. 1 Release profile of propranolol batches made with various PEO at 1:1 drug/polymer ratio, containing $1 \% w / w$ sodium metabisulphite and stored at $40{ }^{\circ} \mathrm{C}$. a PEO 750/303 without sodium metabisulphite. b PEO 750 with sodium metabisulphite. c PEO 303 with sodium metabisulphite

compared to the samples without SMB (compare Fig. 1a with $\mathrm{b}$ and $\mathrm{c})$.

On the basis of above information, some of the features in the dissolution rates of PEO can be elaborated particularly by similarity factor $\left(f_{2}\right)$ values. All $f_{2}$ values for PEO 750 (low molecular weight) and PEO 303 (high molecular weight) are reported to be greater than 50 when the fresh tablets were compared to aged polyox matrices which is an indication of good similarity between their release profiles (Table 2).

Table 3 shows the results of fitting the dissolution data with the dissolution criteria carried out by calculation DE and MDT as described by Khan [31]. As can be seen from Table 3, dissolution efficiency values are reliable with dissolution profiles and these data showed that there was no significant difference between DE data from both PEO 750 and 303. The results obtained for MDT confirmed the same trend (with DE) for both low- and high-molecular-weight PEO, and no major difference was obtained for MDT during the storage of the samples (Table 3).This again verified a stable release rate for both molecular weights PEO in the presence of the antioxidant. For illustration, the dissolution efficiency value of PEO 750 at time 0 was $84.0 \%$ whereas this value decreased to $82.0 \%$ for tablet matrices stored for 8 weeks at $40{ }^{\circ} \mathrm{C}$. Slightly different pattern was obtained for PEO 303 with DE of $57 \%$ at 0 and the same value at week 8 (Table 3 ).

The Evaluation Effect of Sodium Metabisulphite at Ratio $(1 \% w / w)$ on Release Rate Behaviour of Zonisamide and Theophylline Tablet Matrices

In order to cover a wide range of drug solubility, zonisamide, a very poorly water soluble drug $(0.8 \mathrm{mg} / \mathrm{ml})$ and theophylline, a semi-soluble drug $(8 \mathrm{mg} / \mathrm{ml})$ were chosen. The dissolution results of zonisamide and theophylline from the PEO matrix tablets in presence of $1 \%(w / w)$ SMB are depicted in Figs. 2 and 3. SMB readily reacts with dissolved oxygen and is converted into sodium hydrogen sulphate [33]. The formulations containing SMB with both PEO 750 and 303 showed entirely different release profiles compared to the matrices without any antioxidant. For fresh tablets, within the first $2 \mathrm{~h}$, about 90 and $75 \%$ of the drug released from PEO 750 and PEO 303 matrices, respectively, higher than matrices without SMB (compare Figs. $2 \mathrm{a}$ with $\mathrm{b}$ and $\mathrm{c}$ ). At 8 weeks, drug release was further stalled, with approximately $90 \%$ being released after $12 \mathrm{~h}$ in a formulation including PEO 303; however, with time, instead of increasing, the dissolution decreased slightly. A deceleration of drug release at longer storage time is evident in Fig. 2b, $\mathrm{c}$ for preparations containing sodium metabisulphite; however, no major fluctuations can be seen with the antioxidant, proposing that sodium metabisulphite addition has stabilised the oxidation of the polymer. However, as the release profile was getting slower with time, there may be problems with a reproducible in vivo profile.

The difference in the effect of antioxidant is visible in Figs. 2 and 3 compared to that of Fig. 1, with whereby SMB dramatically increases dissolution at week 0 to almost 90 and

Table $2 f 2$ values of propranolol/theophylline/zonisamide-polyox tablet matrices containing $1 \% \mathrm{w} / \mathrm{w}$ concentration sodium metabisulphite at different storage times (2, 4 and 8 weeks)

\begin{tabular}{lllll}
\hline $\begin{array}{l}\text { PEO } \\
\text { grade) }\end{array}$ & $\begin{array}{l}\text { Storage time } \\
\text { (weeks) }\end{array}$ & $\begin{array}{l}\text { 1\% SMB } \\
\text { propanolol }\end{array}$ & $\begin{array}{l}\text { 1\% SMB } \\
\text { theophylline }\end{array}$ & $\begin{array}{l}\text { 1\% SMB } \\
\text { zonisamide }\end{array}$ \\
\hline 750 & 2 & 67.0 & 60.0 & 46.8 \\
750 & 4 & 56.7 & 39.0 & 37.3 \\
750 & 8 & 69.3 & 36.0 & 35.4 \\
303 & 2 & 60.9 & 68.1 & 69.5 \\
303 & 4 & 57.6 & 70.3 & 37.0 \\
303 & 8 & 70.0 & 64.4 & 34.2 \\
\hline
\end{tabular}

Time zero was used as a control 
Table 3 Effect of sodium metabisulphite on dissolution parameters of propranolol/theophylline/zonisamide PEO tablet matrices

\begin{tabular}{|c|c|c|c|c|c|c|c|}
\hline PEO (grade) & Time (week) & $\begin{array}{l}\text { DE }(\%) \\
\text { Propranolol }\end{array}$ & $\begin{array}{l}\text { MDT(h) } \\
\text { Propranolol }\end{array}$ & $\begin{array}{l}\mathrm{DE}(\%) \\
\text { Theophylline }\end{array}$ & $\begin{array}{l}\text { MDT (h) } \\
\text { Theophylline }\end{array}$ & $\begin{array}{l}\mathrm{DE}(\%) \\
\text { Zonisamide }\end{array}$ & $\begin{array}{l}\text { MDT (h) } \\
\text { Zonisamide }\end{array}$ \\
\hline 750 & 0 & 84.0 & 1.17 & 88.0 & 1.06 & 89.0 & 1.11 \\
\hline 750 & 2 & 79.0 & 1.27 & 86.0 & 1.17 & 85.0 & 1.07 \\
\hline 750 & 4 & 81.0 & 1.09 & 84.0 & 1.25 & 84.5 & 1.15 \\
\hline 750 & 8 & 82.0 & 1.33 & 83.0 & 1.36 & 84.1 & 1.25 \\
\hline 303 & 0 & 57.0 & 0.82 & 81.0 & 1.20 & 85.0 & 1.46 \\
\hline 303 & 2 & 61.0 & 0.93 & 79.0 & 1.19 & 84.0 & 1.35 \\
\hline 303 & 4 & 61.5 & 0.85 & 79.0 & 1.21 & 78.0 & 1.44 \\
\hline 303 & 8 & 57.0 & 0.91 & 78.5 & 1.13 & 73.0 & 1.43 \\
\hline
\end{tabular}

$D E$ dissolution efficiency, $M D T$ mean dissolution time

$75 \%$ within $2 \mathrm{~h}$ for PEO 750 and 303, respectively. In contrast, a sustained release was achieved by SMB incorporation in propranolol $\mathrm{HCl}$ polymer formulations. The probable reason for this increased drug release could be the ionic property and solubility of SMB. The presence of sodium ions in the dosage form itself was reported to cause a rapid disintegration of
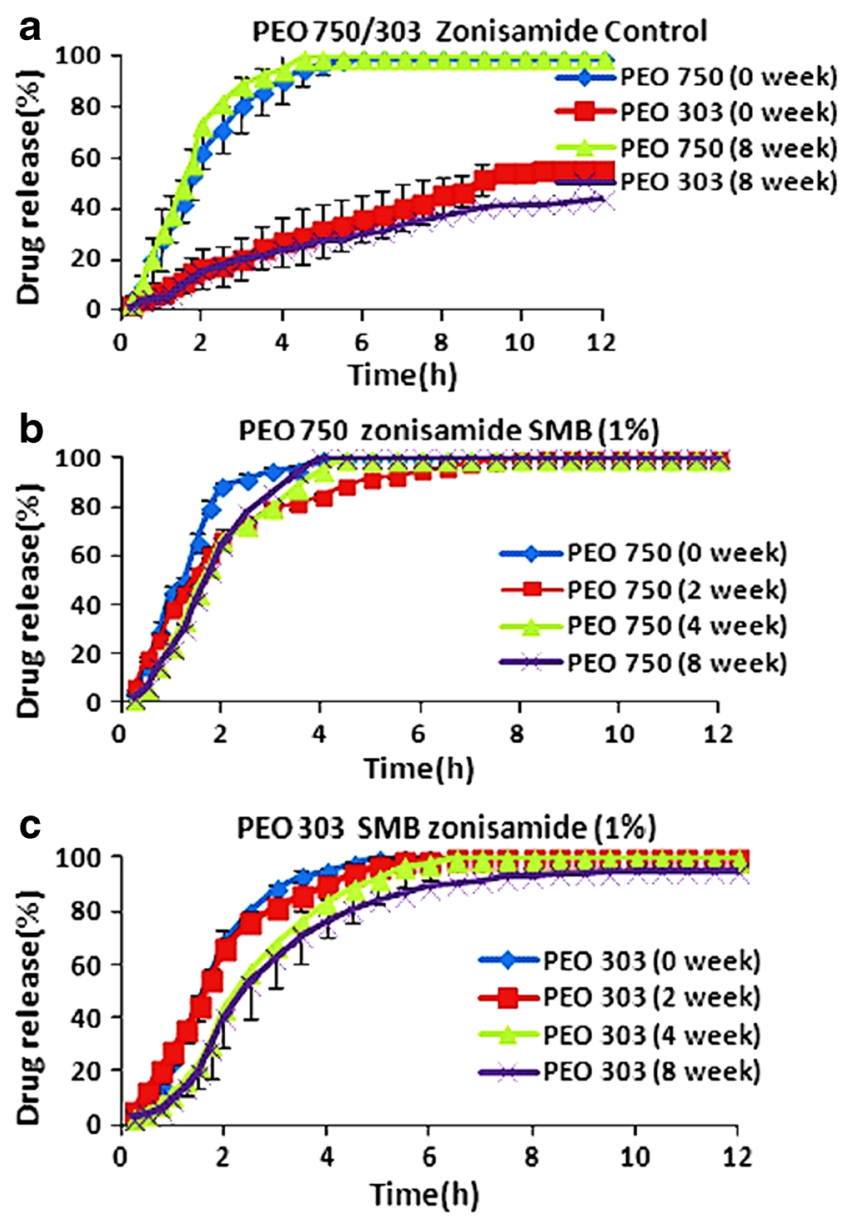

Fig. 2 Release profile of zonisamide batches made with various PEO at $1: 1 \mathrm{drug} /$ polymer ratio, containing $1 \% \mathrm{w} / \mathrm{w}$ sodium metabisulphite and stored at $40{ }^{\circ} \mathrm{C}$. a PEO $750 / 303$ without sodium metabisulphite. b PEO 750 with sodium metabisulphite. c PEO 303 with sodium metabisulphite extended release HPMC matrix tablets [34]. Sodium metabisulphite is readily soluble in water, and in the presence of water, the compound dissociates and forms sodium ion $\left(\mathrm{Na}^{+}\right)$and hydrogen sulphite $\left(\mathrm{HSO}_{3}{ }^{-}\right)$[33]. Higher affinity between sodium ion and water molecules may have facilitated the swelling of matrix quickly. It is suggested that the possible
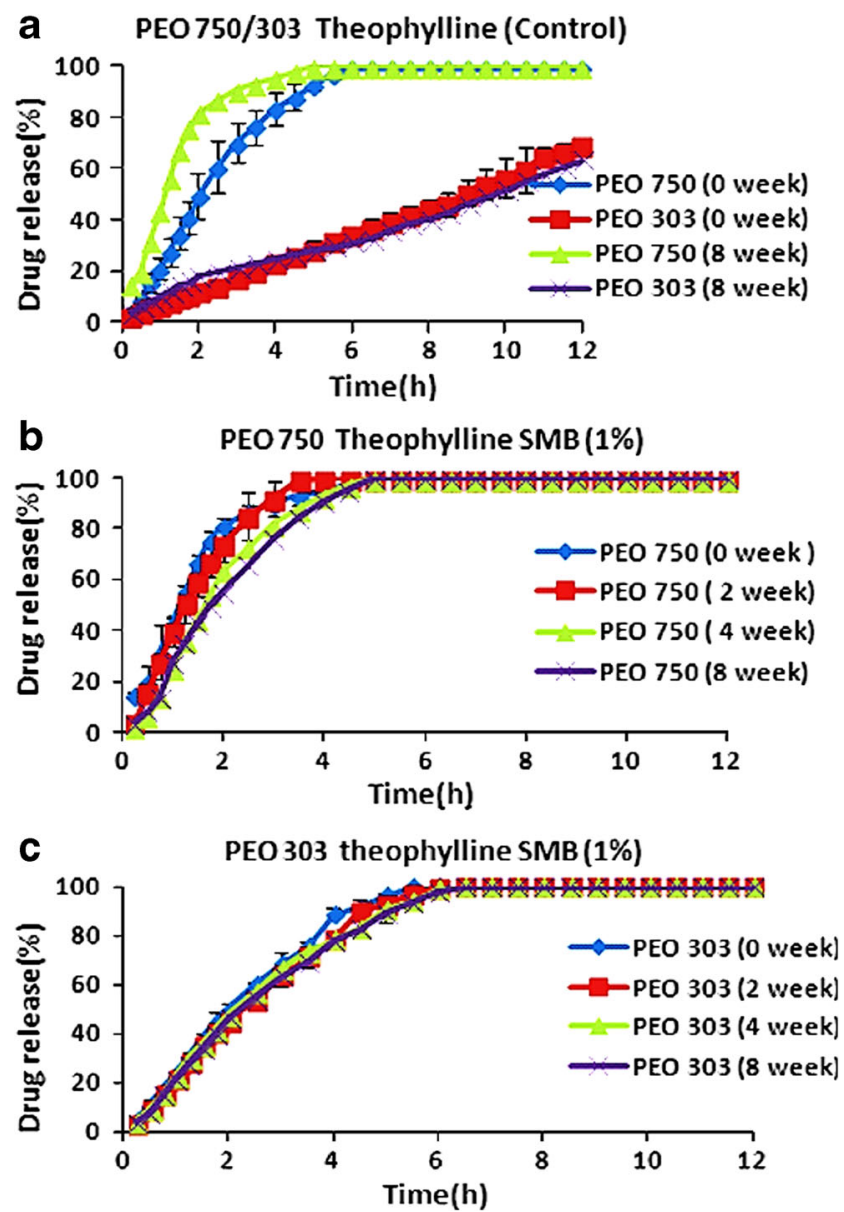

Fig. 3 Release profile of theophylline batches made with various PEO at $1: 1 \mathrm{drug} /$ polymer ratio, containing $1 \% \mathrm{w} / \mathrm{w}$ sodium metabisulphite and stored at $40{ }^{\circ} \mathrm{C}$. a PEO $750 / 303$ without sodium metabisulphite. b PEO 750 with sodium metabisulphite. c PEO 303 with sodium metabisulphite 
enhanced drug release exhibited with sodium metabisulphite is as a result of its high ionic strength, implicating the cloud point (gelation temperature) of gel layers and affecting their formation. The higher the ionic strength, the greater the number of counter-ions available for ion exchange, increasing drug release through enhanced drug unloading [35]. Regardless of the small quantity of sodium metabisulphite present within the batches, the interacting ions may be enough to affect gel layer function, inducing an insufficient barrier and drug release to considerably differ from SMB and the control batch (Fig. 2b, c). It is interesting to note that a good similar pattern release rate was obtained in a formulation containing semi-soluble drug theophylline with both PEO 750 and 303, and the results are shown in Fig. 3b, c. The reason for this drug release was driven for zonisamide tablet matrices earlier in this study. Although, decreasing in release rate with progressive storage time was not noteworthy in theophylline matrices with PEO 303 (Fig. 3c).

To confirm the dissolution profiles, $f_{2}$ test was employed to observe the effect of the antioxidants and results presented in Table 2 shows that all $f_{2}$ values (except for PEO 303 at week 2 , $f_{2}=69.5$ ) are less than 50 , which is determined that there were no similarity between fresh and aged samples, indicating slower drug release at longer storage time in samples containing sodium metabisulphite (Table 2). As can be seen from Table $2, f_{2}$ values for PEO 750 and theophylline were in the same line, although all $f_{2}$ values were obtained for theophylline and PEO 303 are bigger than 50, which demonstrated similar release rate for fresh and aged samples.

DE and MDT as described by Khan [31] for zonisamide and theophylline matrices were carried out to match up to the dissolution data and results are given in Table 3. Dissolution efficiency values confirmed that the drug release rate from various PEO was slower when storage time increased, and a smaller DE was obtained at longer storage time. For instance, the dissolution efficiency value of PEO 750 with theophylline and zonisamide and at time 0 was 88.0 and $89.0 \%$ while this value declined to 83 and $84.1 \%$, respectively, for aged matrices stored for 8 weeks at $40{ }^{\circ} \mathrm{C}$. Similar patterns were observed for the other molecular weight PEO 303 (Table 3). The results obtained for MDT showed a similar trend for theophylline and zonisamide with fresh PEO 750 tablets being 1.06 and $1.11 \mathrm{~h}$ while this value increased to 1.36 and $1.25 \mathrm{~h}$, respectively, at 8-week storage time which is an indication of slower drug release for the tablets stored at $40{ }^{\circ} \mathrm{C}$ for 8 weeks (Table 3).

\section{Kinetics Study}

In order to evaluate the effect of sodium metabisulphite on the mechanism of drug release from the formulations at different storage times, all release data were plotted in Korsmeyer and Peppas equation (power law model) which is the best equation to study the in vitro drug release in such polymeric matrices $[28,29]$. Although the sample preparations may follow zeroorder kinetic, first-order or Higuchi's model, the results of kinetic release obtained from the matrices with and without SMB are given in Tables 4 and 5. The results showed that the $n$ value for both PEO750 and 303 are between 0.68 and 1.49 which is indicated of anomalous release kinetics and indicates a super case II for all different formulations shown in Tables 4 and 5 .

\section{DSC Analysis}

The thermal transitions of the formulations containing SMB before and after storage were investigated by DSC, and results are shown in Figs. 4 and 5 and Tables 6 and 7. For propranolol, the DSC traces of polyox matrices (PEO 750 and 303) with and without SMB clearly showed that there is a significant shift towards higher temperature when metabisulphite was present in the PEO 750 and 303 samples. This means that using the antioxidant could prevent degradation of PEO during a longer storage time with increased temperature. These results obtained via the DSC are summarised in Table 6 including the enthalpy, onset and melting peak. It appears from the table that for samples containing both PEO 750 and 303, the enthalpy values and temperature of melting peak did not decrease as the storage time progressed, maintaining almost similar enthalpies and melting peaks (Table 6).

The DSC traces for zonisamide matrices are presented in Fig. 5 and Table 7. DSC thermograms (Fig. 5) showed that presence of the SMB in PEO 750 and 303 polymer matrices resulted in moving towards higher temperature at longer

Table 4 Effect of storage time on mechanism of drug release of various soluble drugs in absence and presence of $1 \% \mathrm{w} / \mathrm{w}$ from PEO 750 matrices

\begin{tabular}{lll}
\hline Formulation & $r^{2}$ & Number \\
\hline Propranolol HCl & & \\
PEO 750 (fresh) & 0.97 & 0.81 \\
PEO 750 (8 weeks) & 0.96 & 1.29 \\
PEO 750 with sodium metabisulphite (1\% fresh) & 0.93 & 1.29 \\
PEO 750 with sodium metabisulphite (1\%,8 weeks) & 0.95 & 1.24 \\
Theophylline & & \\
PEO 750 (fresh) & 0.99 & 1.11 \\
PEO 750 (8 weeks) & 0.98 & 0.87 \\
PEO 750 with sodium metabisulphite (1\% fresh) & 0.98 & 0.87 \\
PEO 750 with sodium metabisulphite (1\%,8 weeks) & 0.99 & 1.21 \\
Zonisamide & & \\
PEO 750 (fresh) & 0.92 & 1.15 \\
PEO 750 (8 weeks) & 0.97 & 1.86 \\
PEO 750 with sodium metabisulphite (1\% fresh) & 0.98 & 1.44 \\
PEO 750 with sodium metabisulphite (1\%,8 weeks) & 0.99 & 1.49 \\
\hline
\end{tabular}


storage time and an increase in enthalpies as the storage duration increased. This means the fast release rate resultant of using sodium metabisulphite in both PEO 750/303 at week 0 was controlled at longer storage conditions. It is also evident from Table 7 that there was an increase in the melting peak of matrices containing sodium metabisulphite, and then, it was controlled as storage time increased. A quite similar trend for thermal behaviour for theophylline matrices was observed (data not shown).

\section{General Discussion}

To cover a good range of drug solubility in presence of SMB, three model soluble drugs, namely, zonisamide, propranolol $\mathrm{HCl}$ and theophylline, with water solubility of $0.8,60$ and $8 \mathrm{mg} / \mathrm{ml}$, respectively, were chosen. The results showed that the solubility of the drug by itself directly impacted on the release rate of different types of drugs. Moreover, results clearly demonstrated that sodium metabisulphite is a good candidate to prevent degradation in different molecular weight PEO in highly soluble drugs such as propranolol $\mathrm{HCl}(60 \mathrm{mg} / \mathrm{ml})$. The observed results may be deemed expected, according to previous reports by Shojaee et al. [32], and our data for both PEO 750 and 303 containing propranolol $\mathrm{HCl}$ showed faster release for aged tablets without sodium metabisulphite; on the other hand, drug release was controlled in the presence of this antioxidant. It is interesting to note that there is no any other previous research that studied the effect of SMB in sustained release matrices. The results of the current study also illustrated that using SMB in the semi- and poorly water-soluble drugs, such as theophylline and zonisamide, had completely different effect in the beginning of drug release in terms of stability in comparison with the fresh samples without this

Table 5 Effect of storage time on mechanism of drug release of various soluble drugs in absence and presence of $1 \% w / w$ sodium metabisulphite from PEO 303 matrices

\begin{tabular}{lll}
\hline Formulation & $r^{2}$ & Number \\
\hline Propranolol HCl/ PEO 303 (fresh) & 0.99 & 1.05 \\
PEO 303 (8 weeks) & 0.99 & 0.68 \\
PEO 303 with sodium metabisulphite (1\% fresh) & 0.81 & 0.99 \\
PEO 303 with sodium metabisulphite (1\%, 8 weeks) & 0.98 & 0.97 \\
Theophylline / PEO 303 (fresh) & 0.99 & 1.04 \\
PEO 303 (8 weeks) & 0.99 & 1.08 \\
PEO 303 with sodium metabisulphite (1\% fresh) & 0.99 & 1.04 \\
PEO 303 with sodium metabisulphite (1\%, 8 weeks) & 0.98 & 1.06 \\
Zonisamide/ PEO 303 (fresh) & 0.99 & 0.82 \\
PEO 303 (8 weeks) & 0.97 & 0.87 \\
PEO 303 with sodium metabisulphite (1\% fresh) & 0.99 & 1.44 \\
PEO 303 with sodium metabisulphite (1\%,8 weeks) & 0.97 & 1.27 \\
\hline
\end{tabular}

a
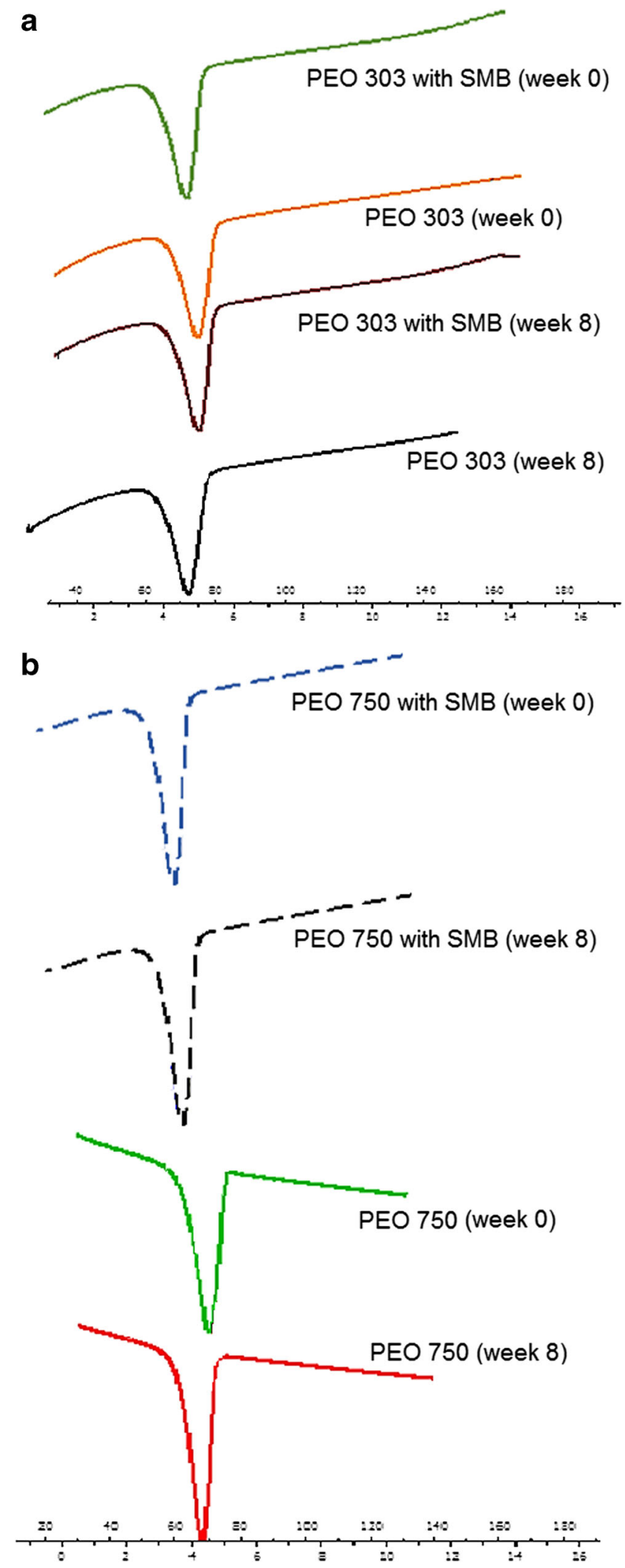

Fig. 4 DSC thermograms of propranolol-PEO 750 and 303 at 8-week storage time without and containing $1 \% \mathrm{w} / \mathrm{w}$ sodium metabisulphite 
Fig. 5 DSC thermograms for zonisamide ground matrix tablets, PEO 750 and 303 with concentration (1\%) sodium metabisulphite, fresh and stored for 8 weeks at $40{ }^{\circ} \mathrm{C}$
PEO 750/ Zonisamide with SMB

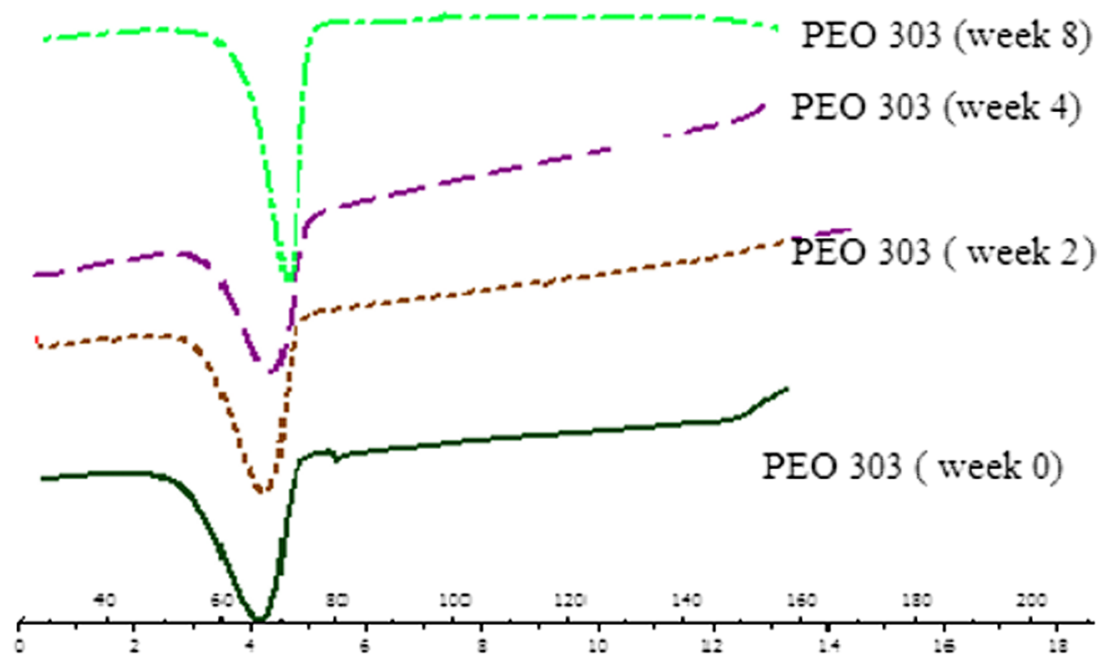

\section{PEO 303/ Zonisamide with SMB}

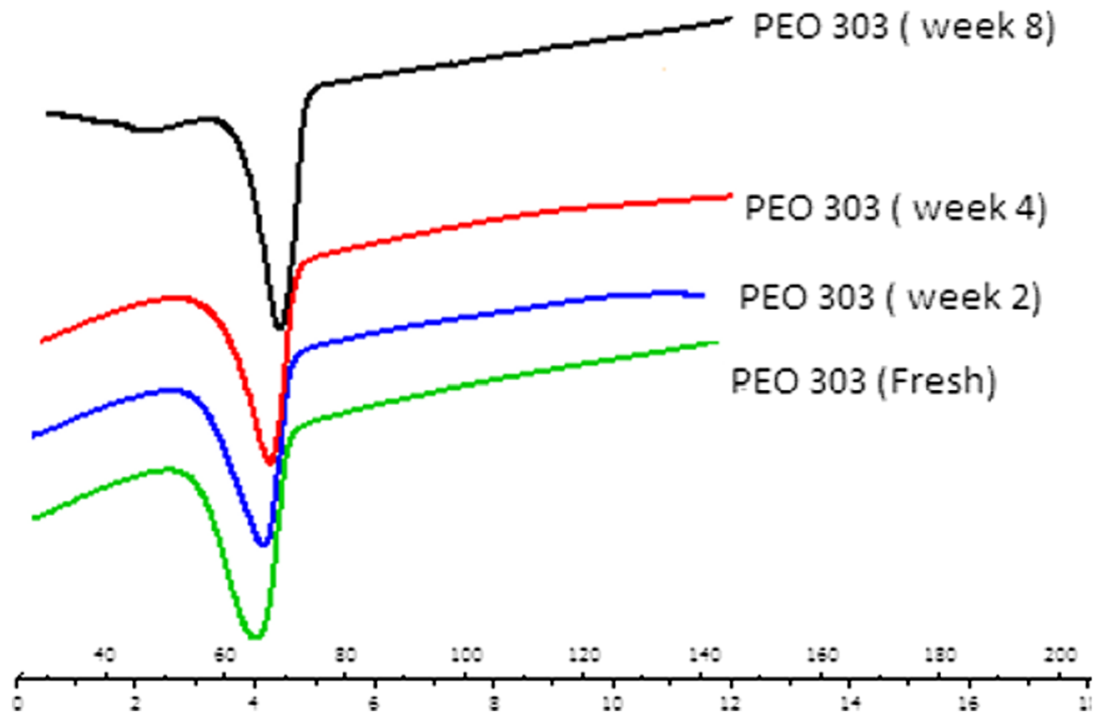

antioxidant, although drug release decreased as storage time increased. These results particularly are not correlated with the previous data published by Shojaee et al. [32,36], who studied the role of sodium metabisulphite on the release rate of diltiazem hydrochloride from polyethylene oxide. This was also a disagreement with earlier data observed in the beginning of this study with propranolol $\mathrm{HCl}$ and the reason is given earlier.
Table 6 Effect of sodium metabisulphite at $1 \% \mathrm{w} / \mathrm{w}$ on thermal behaviour of propranololPEO samples 750 and 303 samples

\begin{tabular}{llll}
\hline PEO samples & Enthalpy $(\mathrm{J} / \mathrm{g})$ & Onset $\left({ }^{\circ} \mathrm{C}\right)$ & Melting peak $\left({ }^{\circ} \mathrm{C}\right)$ \\
\hline 750 fresh & $-135.0 \pm 1.0$ & $64.0 \pm 1.0$ & $71.0 \pm 0.5$ \\
750 (week 8) & $-115.0 \pm 0.2$ & $60.0 \pm 0.5$ & $67.5 \pm 0.1$ \\
750 with 1\% SMB (week 8) & $-130.2 \pm 0.1$ & $62.0 \pm 0.1$ & $70.0 \pm 1.0$ \\
303 fresh & $-165.4 \pm 1.0$ & $64.0 \pm 0.4$ & $71.5 \pm 0.1$ \\
303 (week 8) & $-131.5 \pm 0.4$ & $62.0 \pm 1.0$ & $68.0 \pm 0.3$ \\
303 with 1\% SMB (week 8) & $-161.0 \pm 0.6$ & $63.0 \pm 0.2$ & $71.0 \pm 0.5$ \\
\hline
\end{tabular}


Table 7 Effect of sodium metabisulphite at $1 \% \mathrm{w} / \mathrm{w}$ concentration on thermal behaviour of zonisamide-PEO 750 and 303 samples

\begin{tabular}{llll}
\hline PEO SMB $(1 \%$ w/w $)$ & Enthalpy $(\mathrm{J} / \mathrm{g})$ & Onset $\left({ }^{\circ} \mathrm{C}\right)$ & Melting peak $\left({ }^{\circ} \mathrm{C}\right)$ \\
\hline PEO 303 SMB (week 0) & $-143.0 \pm 1.0$ & $64.0 \pm 0.5$ & $67.0 \pm 0.5$ \\
SMB (week 2) & $-144.0 \pm 0.5$ & $62.0 \pm 0.1$ & $67.5 \pm 0.1$ \\
SMB (week 4) & $-152.5 \pm 0.2$ & $63.0 \pm 1.0$ & $68.7 \pm 0.4$ \\
SMB (week 8) & $-157.3 \pm 0.3$ & $63.0 \pm 0.3$ & $69.0 \pm 0.2$ \\
PEO 750 SMB (week 0) & $-117.0 \pm 1.0$ & $64.0 \pm 0.5$ & $66.0 \pm 0.5$ \\
SMB (week 2) & $-121.0 \pm 0.5$ & $62.0 \pm 0.1$ & $66.5 \pm 0.1$ \\
SMB (week 4) & $-125.5 \pm 0.2$ & $63.0 \pm 1.0$ & $67.1 \pm 0.4$ \\
SMB (week 8) & $-127.3 \pm 0.3$ & $63.0 \pm 0.3$ & $68.0 \pm 0.2$ \\
\hline
\end{tabular}

\section{Conclusions}

The results indicated that the use of sodium metabisulphite marginally assisted in reducing drug release as a result of the minimised degradation via oxidation in propranolol $\mathrm{HCl}$ tablets containing PEO matrices. In the case of poorly and semisoluble drugs (zonisamide and theophylline), the formulations exhibited superimposable phenomenon with different release profiles compared to that of those samples without SMB. The effect of the antioxidant in the tablet formulations is quite evident in the thermal analysis where the presence of SMB seemed to have reduced the thermal degradation of the PEO matrices. Moreover, it was also found that the effect of sodium metabisulphite on the stability and the release of the drugs from the matrix tablets were dependants on both the molecular weight of PEO and the solubility of the drugs used. In conclusion, it can be claimed that overall, the use of an antioxidant in the formulations has made the manufactured tablets stable over 8 weeks of storage.

Open Access This article is distributed under the terms of the Creative Commons Attribution 4.0 International License (http:// creativecommons.org/licenses/by/4.0/), which permits unrestricted use, distribution, and reproduction in any medium, provided you give appropriate credit to the original author(s) and the source, provide a link to the Creative Commons license, and indicate if changes were made.

\section{References}

1. Kiss D, et al. The effect of storage and active ingredient properties on the drug release profile of poly (ethylene oxide) matrix tablets. Carbohydrate Poly. 2008;74:930-3.

2. Khan MZ. Dissolution testing for sustained or controlled release oral dosage forms and correlation with in vivo data: challenges and opportunities. Int J Pharm. 1996;140:131-43.

3. Khan GM. Controlled release oral dosage forms: some recent advantages in matrix type drug delivery systems. The Sciences. 2001:1(5):350-4.

4. Rane M, et al. Hydrophilic matrices for oral extended release. Pharma Times. 2012;42(4):41-5.
5. Maderuelo C, et al. Critical factors in the release of drugs from sustained release hydrophilic matrices. J Control Release. 2011;154:2-19.

6. Davis SS, et al. Transit of pharmaceutical dosage forms through the small intestine. Gut. 1986;27:886-92.

7. Rajabi-Siahboomi AR, et al. Structure and behaviour in hydrophilic matrix sustained release dosage forms. Studies of water mobility and diffusion coefficients in gel layer HPMC tablets using NMR imaging. Pharm Res. 1996;13:376-80.

8. Colombo P, et al. Swellable matrices for controlled drug delivery: gel-layer behaviour, mechanisms and optimal performance. Pharm Sci Technol Today. 2000;3:198-204.

9. Khan MA, et al. Controlled release co-precipitates: formulation considerations. J Con Rel. 1995;37:132-41.

10. Lee PI, et al. Prediction of polymer dissolution in swellable controlled-release systems. J Control Release. 1987;6:207-15.

11. Lapidus H, Lordi NG. Some factors affecting the release of water soluble drugs from compressed hydrophilic matrices. J Pharm Sci. 1966;55:840-3.

12. Wen H, Park K. (2010) Oral controlled release formulation design and drug delivery: theory to practice. New Jersey: John Wiley \& son's Inc. pp. 78-91.

13. Das NG, Das SK. (2003) Controlled-release of oral dosage forms, Formulation, Fill \& Finish pp. 10-16.

14. Li H, et al. Effect of drug solubility on polymer hydration and drug dissolution. AAPS J. 2008;9(2):437-43.

15. Kim CJ. Effects of drug solubility, drug loading and polymer molecular weight on drug release from Polyox tablets. Drug Dev Ind Pharm. 1998;24:645-51.

16. Hongtao L, et al. Effect of drug solubility on polymer hydration and drug dissolution from polyethylene oxide (PEO) matrix tablets. AAPS J. 2008;6:437-43.

17. Huang X, Brazel CS. On the importance and mechanisms of burst release in matrix-controlled drug delivery systems. J Control Release. 2001;73(2-3):121-36.

18. Royce AE. (1993) Directly compressible polyethylene oxide vehicle for preparing therapeutic dosage forms. US Patent 5. 273:758, (Patent).

19. Kim CJ. Drug release from compressed hydrophilic Polyox ${ }^{\circledR}$ WSR tablets. J Pharm Sci. 1995;84(3):303-6.

20. Yang L, et al. A study of the mechanism of the oxidative thermal degradation of poly (ethylene oxide) and poly (propylene oxide) using 1H- and 13C-NMR. Eur Poly J. 1996;32:535-47.

21. Gallet Guillaume G, et al. Thermal degradation of poly (ethylene oxide- propylene oxide- ethylene oxide) triblock copolymer: comparative study by SEC/NMR, SEC/MALDI-TOF-MS and SPME/GC-MS. Poly. 2002;43:1081-94.

22. Dimitrov M, Lambov N. Study of verapamil hydrochloride release from compressed hydrophilic Polyox-WSR tablets. Int J Pharm. 1999;189:105-11. 
23. Maggi L, et al. High molecular weight polyethylene oxides (PEOs) as an alternative to HPMC in controlled release dosage forms. Int J Pharm. 2000;195:229-38.

24. Maggi L, et al. Dissolution behavior of hydrophilic matrix tablets containing two different polyethylene oxides (PEOs) for the controlled release of a water-soluble drug. Biomaterials. 2002;23:1131119.

25. British Pharmacopoeia. (2007) British Pharmacopoeia 2007 volume II general notice, monographs, medicinal and pharmaceutical substances. 5th ed. London: medicines and healthcare products regulatory agency. $1210-1895$.

26. Rowe RC, et al. Handbook of pharmaceutical excipients. London: Pharmaceutical Press and American Pharmacists Association. 2006;38:346-691.

27. USP Pharmacopoeia 26. (2003) National Formulary 21, USP Convention, Rockville, pp. 320.

28. Korsmeyer RW, Peppas NA. Swelling-controlled delivery system for pharmaceutical applications macromolecular and modelling considerations. J Control Release. 1983;15:25-35.
29. Lee PI, Peppas NA. Prediction of polymer dissolution in swellable controlled-release systems. J Control Release. 1987;6:207-15.

30. Moore JW, Flanner HH. Mathematical comparison of dissolution profiles. Pharm Tech. 1996;20:64-74.

31. Khan KA. Concept of dissolution efficiency. J Pharm Sci. 1975;271:48-9.

32. Shojaee $\mathrm{S}$, et al. An investigation into the stabilization of diltiazem $\mathrm{HCl}$ release from matrices made from aged Polyox powders. AAPS PharmSciTech. 2013;14:1190-8.

33. Kasture AV, and Wadodkar SG. (2008) Pharmaceutical Chemistry1, (25th Edition), India:Nirali Prakashan, Educational Publishers, ISBN: 9788185790121 ,

34. Rajabi-Siahboomi AR, et al. Identification of active molecular structures in thermally dependent release behaviour of diclofenac sodium from HPMC matrices. Proc. Int. Syrup. Control. Rel. Bioact. Mater. 1994;21:25-6.

35. Wilson CG, and Crowley PJ. (2011) Controlled release in oral drug delivery, Springer Science New York 10013 USA, pp. 167-170.

36. Kiss D et al. (2008) Tracking the physical ageing of PEO: a technical note, AAPS PharmSciTech. 7: article 95. 